\title{
A case of Kawasaki disease presenting with atypical cutaneous involvement and mimicking Stevens-Johnson syndrome
}

\author{
(D) Ferhat Demir, ${ }^{1}$ (D) Mehtap Haktanir Abul, ${ }^{2}$ (D) Embiya Dilber, ${ }^{3}$ (D) Fazil Orhan, ${ }^{2}$ (iD Mukaddes Kalyoncu ${ }^{1}$ \\ ${ }^{1}$ Department of Pediatric Rheumatology, Karadeniz Technical University Faculty of Medicine, Trabzon, Turkey \\ ${ }^{2}$ Department of Pediatric Allergy and Immunology, Karadeniz Technical University Faculty of Medicine, Trabzon, Turkey \\ ${ }^{3}$ Department of Pediatric Cardiology, Karadeniz Technical University Faculty of Medicine, Trabzon, Turkey
}

\begin{abstract}
Kawasaki disease is the second most common cause of childhood vasculitis. Cutaneous manifestation, which is the diagnostic criteria of Kawasaki disease, may show atypical course and causes conflict in diagnosis for physicians. A 14-month-old girl treated with intravenous immunoglobulin after diagnosed with KD. Target lesions and a vesicular rash developed on the whole body during the course of the disease. Methylprednisolone was added to the treatment. All atypical skin lesions and disease findings were improved using IVIG and subsequent methylprednisolone therapy. Cutaneous signs of KD may show atypical course. Thus, it may lead to confusion in diagnosis with Stevens-Johnson syndrome.
\end{abstract}

Keywords: Kawasaki disease; Stevens-Johnson syndrome; target lesions; vesicular rash.

Cite this article as: Demir F, Haktanir Abul M, Dilber E, Orhan F, Kalyoncu M. A case of Kawasaki disease presenting with atypical cutaneous involvement and mimicking Stevens-Johnson syndrome. North Clin Istanb 2020;7(1):67-70.

$\mathrm{K}^{2}$ awasaki disease $(\mathrm{KD})$ is one of the most common causes of childhood vasculitis. KD was first identified by Tomisaku Kawasaki in Japan. KD may lead to coronary artery disease and death. The etiology has not been explained yet, but some studies suggest that KD is triggered by infections [1]. Cutaneous involvement, which is one of the diagnostic criteria of $\mathrm{KD}$, may show atypical course. The nonspecific, diffuse maculopapular eruption is the most common form of polymorphous rash, but target lesions and vesicular eruptions are rare skin manifestations of the disease $[2,3]$. In this study, we present a case of KD associated with extensive target lesions and vesicular eruptions which may lead to confusion with Stevens-Johnson syndrome (SJS).

\section{CASE REPORT}

A previously healthy 14-month-old female patient admitted to our clinic with a history of fever for four days $\left(\max .40^{\circ} \mathrm{C}\right)$, followed by an extensive rash on the whole body and swelling in the left neck region for two days. Patient consent was obtained for this study. On the first day of high fever, she was admitted to another hospital. Therein, ceftriaxone was administered for three days due to the continued fever and the extensive rash developed on follow up. On admission to our hospital, the patient had fever $\left(38.5^{\circ} \mathrm{C}\right)$ and she was very irritable. Physical examination showed generalized maculopapular erythematous rash on the whole body, erythema in the perineal

Received: March 23, 2018 Accepted: October 10, 2018 Online: December 10, 2018

Correspondence: Dr. Ferhat DEMIR. Karadeniz Teknik Universitesi Tip Fakultesi, Cocuk Romatolojisi Bilim Dali, 61000 Ortahisar, Trabzon, Turkey.

Tel: +90 4623775435 e-mail: drferhat@outlook.com

(c) Copyright 2020 by Istanbul Provincial Directorate of Health - Available online at www.northclinist.com 

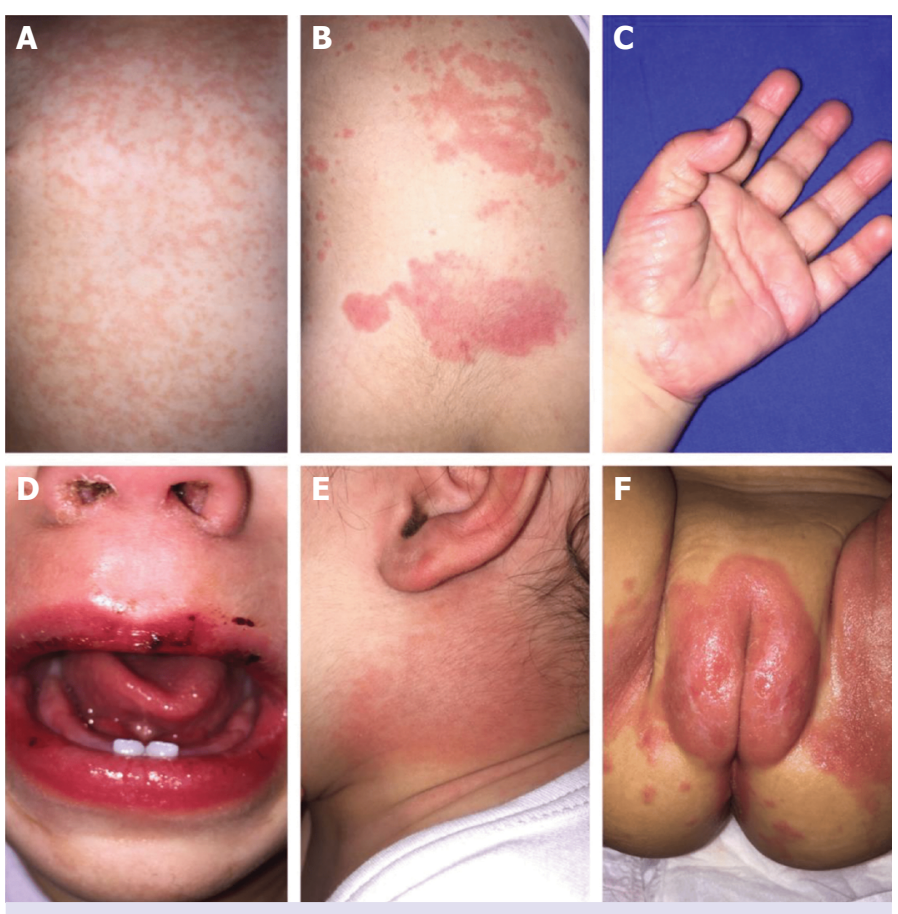

FIGURE 1. Initial cutaneous symptoms; maculopapular erythematous lesions on the body and perineal region (A, B, F), erythema on the left hand (C), crusted lips (D), hyperemic lymphadenopathy in the left cervical region (E).

area, crusted lips, hyperemia in the BCG vaccination side and non-purulent conjunctival congestion. Painful and hyperemic lymphadenopathy $(3 \times 3 \mathrm{~cm}$ India meter) was observed in the left cervical region. She had palmar erythema on both hands, tenderness and swelling in bi- lateral proximal and distal interphalangeal joints (Fig. 1). Laboratory evaluation showed elevated acute phase reactants, including white blood cell count as 41.490/ $\mathrm{mm}^{3}$ with $45.5 \%$ neutrophil and $39.6 \%$ lymphocyte, and platelet count was $508.000 / \mathrm{mm}^{3}$. Liver enzymes were moderately increased. Laboratory results are summarized in Table 1. Infectious work-up that included adenovirus, echovirus, coxsackievirus, Mycoplasma pneumonia, Chlamydia pneumonia, Klebsiella pneumonia, Haemophilus influenzae, influenza (A/B) and parainfluenza were negative. Throat, blood and urine cultures were also negative. Echocardiographic examination of the coronary vessels was normal.

The patient diagnosed as KD and intravenous immunoglobulin (IVIG) $(2 \mathrm{~g} / \mathrm{kg} /$ dose $)$ and acetylsalicylic acid $(80 \mathrm{mg} / \mathrm{kg} /$ day $)$ was started. On the second day of the hospitalization, generalized target lesions were observed. These lesions evolved into the vesicular form on the follow-up, and methylprednisolone $(1 \mathrm{mg} / \mathrm{kg} / \mathrm{day})$ was added to treatment (Fig. 2). The fluid obtained from the vesicular lesions was sterile. The eye examination was carried out by the ophthalmologist and no findings were found except for conjunctival hyperemia. She had fever still on the $3^{\text {rd }}$ day of hospitalization. The second dose IVIG was administered because of the persistent fever. Fever subsided within 24 hours of the second IVIG treatment. Erythematous lesions in the perineal area started to fade, and cracks in the lips and vesicular lesions were crusted four days after the second IVIG therapy. Swelling of the joints recovered on the $7^{\text {th }}$ day. On the

TABLE 1. Laboratory data

\begin{tabular}{|c|c|c|c|c|}
\hline Investigation & On admission & $7^{\text {th }}$ day & At discharge (13 $13^{\text {rd }}$ day) & On follow-up ( $30^{\text {th }}$ day) \\
\hline WBC $(/ \mu \mathrm{L})$ & $41.4 \times 10^{3}$ & $27.1 \times 10^{3}$ & $12.7 \times 10^{3}$ & $8.62 \times 10^{3}$ \\
\hline Neutrophils (\%) & 65.2 & 67.5 & 29.9 & 25 \\
\hline Lymphocytes (\%) & 25.2 & 22.8 & 60.6 & 65 \\
\hline Platelet count $(/ \mu \mathrm{L})$ & $508 \times 10^{3}$ & $943 \times 10^{3}$ & $456 \times 10^{3}$ & $190 \times 10^{3}$ \\
\hline $\operatorname{ESR}(\mathrm{mm} / \mathrm{h})$ & 88 & 37 & 30 & 2 \\
\hline $\mathrm{CRP}(\mathrm{mg} / \mathrm{dL})$ & 23.96 & 6.31 & 1.27 & 0.05 \\
\hline Albumin (g/dL) & 2.4 & 2.7 & 3 & NA \\
\hline Sodium (mEq/L) & 137 & 133 & 135 & NA \\
\hline AST (U/L) & 93 & 26 & 24 & NA \\
\hline $\operatorname{ALT}(\mathrm{U} / \mathrm{L})$ & 51 & 13 & 8 & NA \\
\hline
\end{tabular}

WBC: White blood cell count; ESR: Erythrocyte sedimentation rate; CRP: C-reactive protein; AST: Aspartate aminotransferase; ALT: Alanine aminotransferase; NA: Not available. 



FIGURE 2. Target lesions on the legs at admission (A, B). These lesions evolved to vesicular lesions on the right leg and right arm (C-E). Fading and recovering lesions on the left leg (F).

$8^{\text {th }}$ day of the disease, liver enzymes returned to normal ranges, and platelet count increased to $1.085 .000 / \mathrm{mm}^{3}$. Peeling and desquamation were seen on the $12^{\text {th }}$ day on the fingertips and perineal area. Steroid treatment was stopped within 10 days by tapering. The patient was discharged on the $13^{\text {th }}$ day with acetylsalicylic acid treatment ( $3 \mathrm{mg} / \mathrm{kg} /$ day). On outpatient clinic follow-up, we observed that all the skin and joint symptoms subsided, and acute phase reactants returned to normal ranges (Table 1). Serial echocardiographic evaluation of the coronary arteries did not show any coronary artery abnormality.

\section{DISCUSSION}

Kawasaki disease is an acute self-limited systemic vasculitis that affects medium-sized arteries. Diagnosis is based on clinical features as follows: fever for more than five days accompanied by bilateral conjunctivitis, polymorphous rash, oropharyngeal changes, cervical lymphadenopathy, and peripheral extremity changes. Patients who met four or more clinical criteria together with fever are defined as complete/classical KD. Incomplete KD description is used for cases having a fever with two or three clinical criteria [4]. Our patient was diag- nosed as a complete KD because she had met all the clinical criteria mentioned above. We should note that this case was very attractive because of the development of atypical cutaneous manifestations that included target and vesicular lesions on the follow up.

Cutaneous manifestations are within the diagnostic criteria of KD. It is seen frequently on the whole body as macular and morbilliform skin rashes during the disease course. Erythema of the perineal area is frequently accompanied by morbilliform skin rash. These cutaneous findings rarely show an atypical course like a bullous and vesicular rash. A case of KD associated with vesicles was firstly reported by Stadelman et al. in 1978 [2]. Pustular lesions are one of the other rare skin manifestations of KD. Steril pustular lesions were observed in four of 75 patients diagnosed with KD in a Japan study by Kimura et al. These pustules developed on the $6^{\text {th }}$ day of fever and healed within 7-12 days [5]. Another case of KD, which initially considered as chickenpox, with pustulovesicular lesions was reported in the literature [3]. The psoriasiform lesions were another uncommon cutaneous sign of disease. Eberhard et al. published a series of 10 patients that developed psoriatic skin rash during either acute and convalescent phase of the KD. Skin rashes were found in three patients as pustular and in the remaining seven patients as more typical psoriasiform skin lesions. No chronic psoriasis has developed in any patient [6]. Ming et al. reported three patients with annular lesions as an unusual cutaneous manifestation of KD. In addition, several cases of KD with erythema multiforme were published [7-9]. Vierucci et al. presented a case of KD with erythema multiforme, which was treated by only IVIG and lesions completely resolved after 30 days [9].

Stevens-Johnson syndrome is an immune complexmediated hypersensitivity reaction that typically involves the skin and mucous membranes. In cutaneous involvement, erythematous maculopapular plaques and vesiculobullous lesions are the most common lesions. The typical lesion has a target appearance. The involvement of the eye and mucous membranes of the oral, nasal, vaginal, gastrointestinal and lower respiratory tracts can be seen in the course of the disease. SJS may cause severe morbidities and death. The disease can be triggered by different etiologic causes (infections, drugs, malignancies) [10].

Skin lesions of our patient started as a maculopapular erythematous rash that is typical for KD. Then, it was initially evolved into annular and target lesions on 
the $6^{\text {th }}$ day and into sterile vesicular lesions on the $8^{\text {th }}$ day of the disease, which was more distinct in the extremities. All these lesions gradually fade away within 11 days. Because the lesions developed one day after IVIG treatment, it was also evaluated as a skin reaction due to treatment. It has been seen in the literature review that the most common cutaneous reactions after IVIG treatment are the transient urticaria and erythematous-maculopapular rash, which developed within the first hours of treatment. It has been reported that target lesions do not develop as a post-IVIG skin reaction, occasionally isolated vesicular lesions may occur without erythema, and they are often developed in the hands and feet of the foot [11-13]. IVIG was not considered in the etiology because the skin lesions reported in the literature after IVIG treatment was not compatible with the lesions of our patient. SJS was considered in the differential diagnosis because of the use of other medications in the history and the development of target and vesiculo-pustular lesions in the follow-up. SJS was not considered as the final diagnosis due to the absence of eye involvement, presence of target lesions predominantly in the extremities and the existence of the intact areas between the lesions. With the reason for fulfilling all the diagnostic criteria, the patient was evaluated as KD. Cutaneous signs of KD may show atypical course. Thus, it may lead to confusion in diagnosis. In cases not fulfilling the diagnostic criteria of KD or showing atypical course Stevens-Johnson syndrome, toxic epidermal necrolysis, scarlet fever, measles, other viral exanthems, streptococcic or staphylococcal toxic shock syndrome and systemic-onset juvenile idiopathic arthritis should be considered in the differential diagnosis.

\section{Conclusion}

In conclusion, early diagnosis and treatment of $\mathrm{KD}$ are important for the clinical course and prognosis of the disease. Especially pediatricians and dermatologists should consider KD in the differential diagnosis of each patient with persistent fever even in the patients with unusual skin symptoms, such as target and vesicular lesions. In this study, we presented a case of KD with atypical cutaneous manifestations that could be confused with SJS to emphasize the importance of differential diagnosis.
Informed Consent: Written informed consent was obtained from the patient for the publication of the case report and the accompanying images.

Conflict of Interest: No conflict of interest was declared by the authors.

Financial Disclosure: The authors declared that this study has received no financial support.

Authorship Contributions: Concept - FD, MHA, FO MK; Design FD, MHA, FO, MK; Supervision - FD, MHA, FO, MK; Materials - FD, MHA, ED; Data collection and/or processing - FD, MHA, ED; Analysis and/or interpretation - FD, MHA, ED; Writing - FD, FO, MK; Critical review - FO, MK.

\section{REFERENCES}

1. Burns JC, Glodé MP. Kawasaki syndrome. Lancet 2004;364:533-44.

2. Stadelman PM, Jaouen RM, Wang SF. Mucocutaneous lymph node syndrome with vesicles. J Med Soc N J 1978;75:56-8.

3. Kwan YW, Leung CW. Pustulo-vesicular skin eruption in a child with probable Kawasaki disease. Eur J Pediatr 2005;164:770-1. [CrossRef]

4. Newburger JW, Takahashi M, Gerber MA, Gewitz MH, Tani LY, Burns JC, et al. Diagnosis, treatment, and long-term management of Kawasaki disease: a statement for health professionals from the Committee on Rheumatic Fever, Endocarditis and Kawasaki Disease, Council on Cardiovascular Disease in the Young, American Heart Association. Circulation 2004;110:2747-71. [CrossRef]

5. Kimura T, Miyazawa H, Watanabe K, Moriya T. Small pustules in Kawasaki disease. A clinicopathological study of four patients. Am J Dermatopathol 1988;10:218-23. [CrossRef]

6. Eberhard BA, Sundel RP, Newburger JW, Baker A, Fuhlbrigge RC, Burns JC, et al. Psoriatic eruption in Kawasaki disease. J Pediatr 2000;137:578-80. [CrossRef]

7. Ming A, Wargon O. Annular lesions in Kawasaki disease: a cause of confusion. Australas J Dermatol 2008;49:207-12. [CrossRef]

8. Eun LY, Go JW, Kang WH, Kim SH, Cho HK. Erythema multiforme associated with Kawasaki Disease in a Korean child. Eur J Dermatol 2010;20:524-5. [CrossRef]

9. Vierucci F, Tuoni C, Moscuzza F, Saggese G, Consolini R. Erythema multiforme as first sign of incomplete Kawasaki disease. Ital J Pediatr 2013;39:11. [CrossRef]

10. French LE. Toxic epidermal necrolysis and Stevens Johnson syndrome: our currentunderstanding. Allergol Int 2006;55:9-16. [CrossRef]

11. Hurelbrink CB, Spies JM, Yiannikas C. Significant dermatological side effects of intravenous immunoglobulin. J Clin Neurosci 2013;20:11146. [CrossRef]

12. Orbach H, Katz U, Sherer Y, Shoenfeld Y. Intravenous immunoglobulin: adverse effects and safe administration. Clin Rev Allergy Immunol 2005;29:173-84. [CrossRef]

13. Lin WL, Lin WC, Chang YC, Yang LC, Hung SI, Hong HS, et al. Intravenous immunoglobulin-induced, non-eczematous, vesiculobullous eruptions in Stevens-Johnson syndrome. Am J Clin Dermatol 2009;10:339-42. [CrossRef] 